\title{
ARQUEOLOGÍA DE ÑAUPAS
}

\author{
J. E. González Carré y Carlos Chahud
}

\section{INTRODUCCIÓN}

Naupas es un yacimiento arqueológico ubicado en las cercanías del pueblo de Paqcha, dentro del distrito de Vinchos en el departamento de Ayacucho, sierra central del Perú.

Culturalmente Naupas representa una unidad, por el conjunto de evidencias que hemos encontrado, pero se trata de dos conjuntos cercanos de estructuras que nos demuestran haber sido parte integrante de una población esparcida depositaria de una tradición cultural común.

Desde la ciudad de Ayacucho existe comunicación por carretera con el distrito de Vinchos, pero a partir de este lugar se debe utilizar un camino de herradura para llegar a Paqcha pudiendo hacerse el viaje a pie o en acémila durante horas.

Paqcha es un pueblo etimológicamente importante por tratarse de un grupo humano cuyas costumbres difieren notablemente de sus similares que se encuentran en el área teniendo poco contacto con la ciudad de Ayacucho y desarrollar una vida de aislamiento voluntario frente al avance de la modernización.

El pueblo de Paqcha se encuentra aproximadamente a unos $15 \mathrm{Km}$. al nor este del pueblo de Vinchos y tiene una altitud de 3500 m.s.n.m.

La unidad arqueológica que hemos denominado Naupas, se compone de dos sitios co- nocidos como Atún Ñaupas y Uchuy Ñaupas, nombres dados por los mismos lugareños y que aluden al tamaño y antigüedad de cada uno de los conjuntos. Naupas se refiere al que se encuentra "adelante" o mas "antiguo"; Atún sirve para denominar un lugar extenso y Uchuy a un sitio pequeño y reducido.

Hatun Naupas se encuentra a unos $8 \mathrm{Kms}$. Al nor este de Paqcha, siendo visible desde este pueblo. Es un cerro cuya, altura es de 3,700 m.s.n.m. Las faldearías de este cerro, presentan en sus cuatro lados gran cantidad de enormes rocas lo que dificulta su acceso. Limita al O con la gran quebrada de Hatun Naupas Wayqu; al S.O. con Quchapata; akl Sur con Lliwa; al E. con Muqupampa; al S.E. con la casa comunal de Chichirayuq y con $\mathrm{Cu}$ rachayoq Muq; al N:O con Aqumarca Qasa y al Norte con Qarwachayoq.

Uchuy Naupas, ubicado al E. de Paqcha y muy cerca de Huatun Naupas. Limita al N. con Naupa Chaka; al N.O. con Hatun Naupas; al Sur con el cerro Antasol; por el ÇO. Con Paqcha Waiju y por el E. con el río Naupa Chaka.

\section{LAS ESTRUCTURAS}

Hatun Naupas presenta un conjunto de habitaciones, en número aproximado de 400 , en 
buen estado de conservación y que albergaría a una considerable población.

Las casas son de forma circular, unas son más grandes que otras y el conjunto no responde a una clara planificación, teniendo evidentes semejanzas a los sitios de Pilluco y Aukimarca en la Hoya del río Pampas en la región Cangallo, Departamento de Ayacucho.

Para su construcción se han empleado piedras de forma irregular, colocadas formando un parejo constituido de dos hileras de piedras unidas con argamasa de barro en algunos casos. El ancho de los muros varia entre 0.40 y $0.70 \mathrm{cms}$. el paramento no tiene revoque y las piedras presentan aristas y salientes.

No se han encontrado evidencias de los materiales y la forma del techo que utilizaron en estas construcciones circulares, pero es posible que haya sido la forma cónica y el material básico habría sido la paja natural de las zonas altas conocidas como Ichu; ya que actualmente, los grupos de pastores q2ue viven en las zonas altas de la región continúan construyendo en forma circular y utilizando el tipo de techo que mencionamos.

Las casas están dispuestas sin ningún orden y las distancias entre una y otras presentan variaciones de $4 \mathrm{mts}$., $3 \mathrm{mts}$.; $2.50 \mathrm{mts}$. y $1 \mathrm{~m}$.; presentándose casos en que dos construcciones se encuentren completamente unidas.

Como hemos dicho, las construcciones, en general, pueden ser tipificadas como circulares a primera vista, pero presentan ligeras deformaciones que dan lugar a que no formen un círculo perfecto, para adecuarse a los accidentes del terreno.

Tomando aproximadamente el centro, hemos constatado que se presentan las siguientes variaciones en las dimensiones: 3.35 x $2.60 \mathrm{mts}$. de radio; 3.90 x $3.90 \mathrm{mts}$. De radio, plenamente circular, 4.70 x $4.20 \mathrm{mts}$. De radio y 6.10 x 5.50 mts. De radio.

Los vanos de acceso, en una gran mayoría, no tienen muy notoria su exacta ubicación por encontrarse totalmente derruida la parte de los muros donde estaban ubicados, Pero en los casos que se han podido comprobar la entrada se encuentra ubicada al norte.

En Uchuy Naupas las construcciones que pueden ser identificadas son pocas en numero y no llegan a un centenar las que se encuentran en buenas condiciones de conservación, ya que la mayoría se encuentra derruidas debido a que el terreno es utilizado para realizar sombríos de cebada.

Uchuy Ñaupas es relativamente más pequeño, pero se debe tener en cuenta la gran cantidad de muros derruidos cuyos materiales han sido utilizados en la construcción de cercos para corrales contemporáneos. La destrucción y los restos de estructuras que existen en el lugar hacen difícil precisas el numero de habitaciones con exactitud sin haber realizado previamente una limpieza en el sector.

Las características arquitectónicas y los principios de organización de las edificaciones de Uchuy Naupas son totalmente similares a las que se presentan en Hatun Naupa lo que nos lleva a pensar que ambos sitio eran simplemente dos sectores de un solo conjunto poblacional.

Las habitaciones siguen siendo circulares, tanto el aparejo como el paramento de los muros tienen similares características al Hatun Naupas. El espesor de los muros varia entre 0.50 y 0.68 cms., el radio de las habitaciones varia entre $5.70,6.50$ y $3.50 \mathrm{mts}$. E radio tomado a partir del centro de las habitaciones.

En ambos sectores no ha sido posible precisar con exactitud la altura de los muros por no encontrarse completos, pero se encuentra una variación entre las medidas tomadas que va de $70 \mathrm{~cm}$. a $1.32 \mathrm{mts}$. de altura y el anchos de las puertas también varia entre $86 \mathrm{cms}$. A $1.32 \mathrm{mts}$. en ambos sectores.

Los sitios que describimos se encuentran en lugares estratégicos, en alturas desde las cuales es posible observar amplios sectores. En las falderías y pendientes que rodean ambos sitios se encuentran restos de lo que podrían ser tipificadas como una arquitectura funeraria de características muy peculiares que desarrollaron estos grupos humanos. 
En las falderías este un conjunto de abrigos rocosos naturales que ha sido aprovechados como lugares de enterramientos múltiples. En estos abrigos se ha construido 'pequeños muros a manera de contención en unos casos y en otros en forma de nichos de gran tamaño en el interior de los cuales se encuentran gran cantidad de restos humanos con diferentes deformaciones, todo ello asociados con restos de cerámica fragmentada.

\section{LA CERAMICA}

La exploración superficial realizada nos ha permitido recolectar un pequeño conjunto de 4 fragmentos en toda el área del yacimiento de Naupas en número de 350. Tomando como base esta pequeña muestra superficial hemos intentado una clasificación preliminar logrando diferencias los siguientes tipos:

1. Ñaupas Rojo Pintado.

2. Naupas Naranja alisado.

3. Naupas Ante

4. Naupas Tosco

5. Naupas Ante Claro

6. Naupas Gris.

\section{1. Ñaupas Rojo Pintado.}

La pasta presenta algunas variaciones por el tipo de desgrasante utilizado, pudiendo observar la utilización de cuarzo en gran proporción; también existe una mezcla de cuarzo y mica de fina apariencia y una tercera variante de combinar mica y cuarzo pero presenta porosidades en a pasta.

El color de la pasta tiene distintas tonalidades que van de un naranja claro al gris, ya que si el conocimiento ha sido por atmósfera oxidante esta se ha realizado en forma totalmente incompleta.

La superficie exterior es de color rojo ligeramente diluido y el tratamiento aplicado es el alisado. El interior de los fragmentos tiene el mismo color rojo pero en algunos casos es de color ante, el tratamiento es también alisado auque en algunos casos se aplica el brochado.
El método de manufactura empleado ha sido el modelado.

Los bordes son de labios aplanados de forma recta, doblados al exterior en algunos casos y ligeramente ensanchados. La abertura de los bordes es de 26, 14 y $18 \mathrm{~cm}$.

El espesor de los fragmentos varía entre 3 y $7 \mathrm{~mm}$ hasta $1 \mathrm{~cm}$. Las asas son cintadas con una hendidura en el centro y su ancho es de 5 y $3 \mathrm{~cm}$. Las bases son planas y mamiformes.

\section{Naupas Naranja Alisado}

La pasta tiene como desgrasante la mica y el cuarzo en cantidades proporcionales, no presenta porosidades y es de una textura regular con apariencia compacta. Su color varía de un ante claro a rojizo y su cocimiento es de oxidación completa.

La superficie exterior es alisada y de color naranja pálido sin ningún tipo de pintura o engobe. El interior de los fragmentos tiene las mismas características. El método de factura es el modelado.

Los bordes son rectos, de labios redondeados y con engrasamiento en algunos casos. Las aberturas varía entre $1014 \mathrm{~cm}$ y su espesor de los fragmentos va desde $7 \mathrm{~mm}$ hasta a $1.5 \mathrm{~cm}$. Las asas son cintadas de 2.3 y $3 \mathrm{~cm}$ de ancho y las bases son mamiformes.

\section{Naupas Ante}

La pasta está compuesta de gran cantidad de cuarzo y algunas incrustaciones de mica con asperezas y porosidades. El color varía de un ante pálido al naranja y gris claro. El cocimiento es de una oxidación incompleta. El método de factura es el modelado.

La superficie exterior es de un alisado irregular, erosionada y deleznable al tacto notándose las partículas de desgrasante. El color es ante claro y oscuro. El interior es de un alisado menos uniforme, también hay fragmentos brochados siendo el color también ante. 
Los bordes son de labios redondeados, de forma recta, en algunos casos ligeramente expandidos al exterior y con pequeño engrosamiento. La abertura es de 2, 18 y $10 \mathrm{~cm}$. El espesor de los tiestos varía entre $1 \mathrm{~cm}$ y $8 \mathrm{~mm}$. Las bases son planas y las asas cintadas, algunas con hendiduras al centro y el ancho varía entre 2 y $4 \mathrm{~cm}$.

Existen dos fragmentos que corresponden a cuellos de vasijas con una decoración de bandas verticales incisos parecidas la decoración de lo que se conoce como Argalla.

\section{Naupa Tosco}

El desgrasante se compone de cuarzo y mica, formando una mezcla áspera y porosa, el color es de varias tonalidades que van de negro a gris. El cocimiento es de una oxidación incompleta. El método de factura es el modelado.

La superficie exterior es de color ante claro con alisamiento irregular notándose el temperante. Interiormente se utiliza el alisado más uniforme y también el brochado.

Los bordes son de labios redondeados y en algunos casos aplanados, ligeramente expandidos y también rectos. La abertura varía entre 18,28 y $30 \mathrm{~cm}$. El espesor de los fragmentos es de $7 \mathrm{~mm}$ hasta los $13 \mathrm{~mm}$.

La base es de tipo mamiforme y las asas cintadas y toscas de 3 y $4 \mathrm{~cm}$ de ancho.

\section{Naupas Ante Claro}

La pasta en algunos casos tiene preponderancia de cuarzo y en otros se presenta en combinación con mica, el color marrón oscuro al negro. La cochura es de una oxidación incompleta y el método de manufactura es el modelado.

Ambas superficies son alisadas y de un color ante muy claro que tiende a un blanco grisáceo.

Los bordes son rectos y ligeramente expandidos, de labios aplanados y redondeados. La abertura varía entre 18 y $24 \mathrm{~cm}$, las bases con mamiformes y el espesor de los tiestos es de 10 y $15 \mathrm{~mm}$. Las asas son cintadas, toscas y su grosor varía entre 3 y $3.5 \mathrm{~cm}$.

\section{Naupas Gris}

La pasta tiene como desgrasante básico al cuarzo con algunas incrustaciones de mica, la mezcla no es homogénea y su color varía de ante a negro y gris, el cocimiento es de oxidación incompleta. El método de manufactura es el modelado.

Exteriormente la superficie es alisada de color gris oscuro en el interior los fragmentos son alisados y también existen algunos brochados.

Los bordes son ligeramente doblados hacia fuera y también expandidos de labios redondeados. Las bases son mamiformes y las asas cintadas, con un ancho que varía los 2 y $5 \mathrm{~cm}$. La abertura de los bordes presenta variaciones que van de los 16 a los $20 \mathrm{~cm}$ y el espesor de los fragmentos tiene 5 y $8 \mathrm{~mm}$.

Todos los fragmentos presentan en la superficie manchas de carbón y restos de partículas de ceniza, tomando coloraciones grises y negras, que son producto de la utilización de los recipientes en tareas domésticas.

\section{MATERIAL LÍTICO}

Hemos recolectado dos tipos de artefactos de piedra de diferente utilización y características. Los artefactos líticos se encuentran fracturados y es posible colectar en el lugar diferentes fragmentos producto de roturas como también pedazos de artefactos que no han sido terminados de elaborar.

Un tipo es el conocido como Maqanas, que son artefactos circulares con un orificio central, evidentemente con un fin de introducir un mango. El espesor de los artefactos varía entre 2.5 y $3 \mathrm{~cm}$ en igual forma que la abertura del orificio central. 


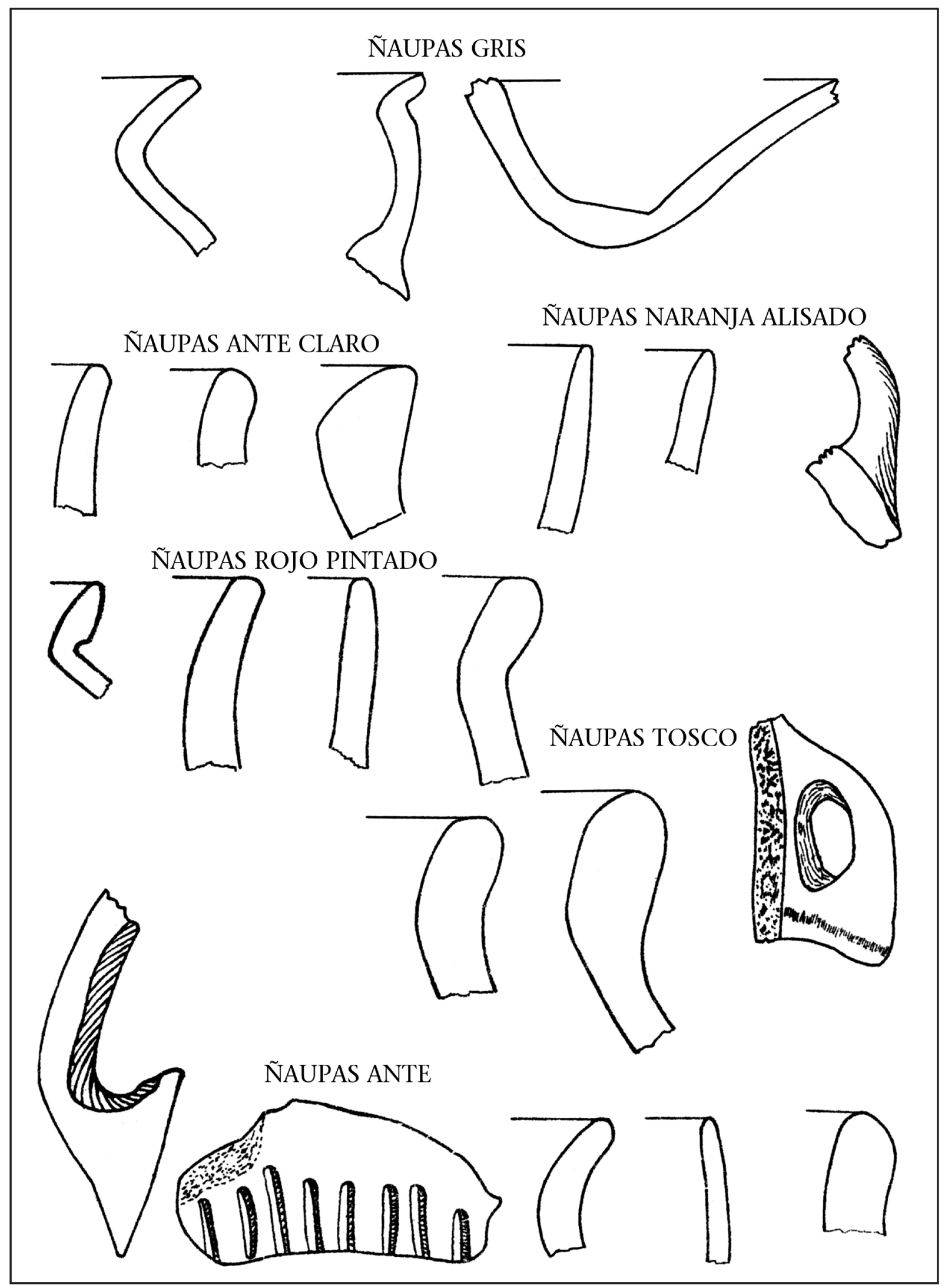


Un segundo tipo, las formas determinadas hachas, que son artefactos laminados que en uno de sus bordes presentan un afilado uniforme. Estas láminas tienen un espesor de $8 \mathrm{~mm}$ y $1 \mathrm{~cm}$.

Ambos artefactos presentan un pulido superficial, conseguido a través de la abrasión.

\section{CONSIDERACIONES GENERALES}

Por el conjunto de evidencias recogidas y las comparaciones con materiales similares de la Sierra Central, el sitio arqueológico de Naupas es un lugar de ocupación tardía que proporcionará mayores elementos para el estudio de lo que hoy, y en forma tentativa, se ha venido identificando culturalmente con el nombre de Chankas.

Naupas debe ser incluido en la lista de sitios arqueológicos correspondiente a la etapa de Estados Regionales o Intermedio Tardío, consignada por Luis Lumbreras en un trabajo al II Congreso Nacional de Historia del Perú (1958); trabajo que es el resultado de sus exploraciones en las hoyas de los ríos Cachi y Pampas en el departamento de Ayacucho.

Por las excavaciones realizadas en Vilcashuamán y en Pillucho durante 1965 por el Instituto de Antropología de la Universidad de Huamanga, se ha comprobado que los materiales que son denominados actualmente como Chankas se encuentran estratégicamente en posición anterior al material cultural reconocido como incaico, a la vez, en algunos casos hay cierta contemporaneidad en la ubicación de lo Chanka con lo Inca. Evidentemente que las evidencias recogidas hasta el momento, permiten con mayor seguridad precisar la ubicación temporal de lo conocido como Chanka.

Por la comparación hecha entre los materiales de Naupas, Vilcashuamán y Pillucho, notamos la existencia de rasgos similares tanto en lo que a cerámica se refiere, como también a los restos de su estructura y material lítico. Esto viene a corroborar lo que Luis Lumbreras anota en su trabajo ya mencionado, al decirnos que "Los Chankas inmediatamente antes que los Incas y contemporáneos con ellos" (1958 p. 228): lo cual es importante para la ubicación en el tiempo de este grupo, ya que si Vilcashuamán es un yacimiento típico incaico y la posición del material Chanka estratigráficamente es anterior y contemporáneo a la vez, la etapa del Intermedio Tardío y los primeros momentos del Horizonte Incaico sería el momento en el que se desarrollaron las manifestaciones Chankas. Esta afirmación no es hipotética pues la hacemos en base a evidencias recogidas en el campo.

En cuanto a la arquitectura y los patrones de asentamiento, las características de las construcciones de Naupas nos permiten establecer relaciones homólogas con los rasgos encontrados en los sitios arqueológicos de Caballoyuq, Pillucho, Arqalla, Ayataki, Lawirasqa, Aukimarca y otros.

En general las construcciones son circulares ubicadas en las cumbres de los cerros y dispuestas en forma desordenada. La ubicación en lugares altos y abruptos obedecería a una estrategia defensiva, tal como lo afirma Duccio Bonavia en su informe sobre sus investigaciones en Caballoyuq: "todos obedecen al mismo patrón de ocupación consistente en construir en las puntas de los cerros, evitando las laderas y las partes bajas. Es una medida estratégica de protección: en determinados lugares hay estructuras de defensa" (1964 p. 51). También es frecuente encontrar en una misma área, construcciones circulares junto con construcciones incaicas, y aún más en la actualidad, este tipo habitacional tiene similitud con el usado entre grupos de pastores andinos contemporáneos.

Los muros circulares que encontramos en Naupas como los otros sitios ya mencionados, coinciden con la descripción de Lumbreras que. Al referirse a la técnica y a los materiales usados anota que "la estructura está dada por piedras de forma irregular, generalmente canteadas y unidas, una veces con barro mientras que en otras es de simple mampostería" (1958, p. 232).

El material lítico procedente de Naupas es característico y está formado por artefactos catalogados por nosotros como hachas y maqanas. Este tipo de hacha es algo diferente a lo conocido hasta el momento como tal. 
Este artefacto $\mathrm{n}$ mención lo ha ubicado Lumbreras en sitios Chankas y nos dice que son "artefactos de piedra achatada en forma de hachas y que algunos creen que han sido instrumentos de labranza y otros hacha" (1958, p. 233).

En lo que se refiere a cerámica hemos logrado diferenciar seis sitios, todos caracterizados por presentar evidencias de una mala manufactura, que si bien no es incipiente, se aleja de todas las técnicas usuales en el área andina que durante esta etapa ya habían alcanzado un alto desarrollo, es decir, es una cerámica tosca en comparación con otras cronológicamente anteriores y posteriores.

Tecnológicamente la cerámica nos demostraría que loa grupos humanos que la fabricaron no contaban con los medios y las condiciones adecuadas para producir una alfarería fina y bien elaborada, teniendo en cuenta que los pueblos andinos dominaban y conocían plenamente la técnica alfarera, presentándose el caso Chanka como una "regresión" en dicha actividad. La razón de este fenómeno debe buscarse no en la cerámica misma sino en las condiciones sociales y económicas que la generaron.

El tipo que hemos denominado Naupas Rojo, presenta similitudes con la descripción que hace Lumbreras de tipos ubicados por él en yacimientos semejantes. "La cerámica pintada tiene como elemento principal un engobe rojo de poca consistencia y diluido. Es un rojo semejante al que se conoce con el nombre de rojo ladrillo. Hay unos cuantos fragmentos con un engobe rojo consistente y bruñido" (1958, p. 234).

Las otras características de las cerámica de Naupas como son: base mamiforme, asas cintadas con hendiduras al centro y decoración incisa, también han sido halladas por Lumbreras en los lugares que él visitó (1958, p. 234).

El tipo que denominamos Naupas Ante, que lo hemos comparado con muestras de Caballoyuq, analizadas por Bonavia, presenta evidente relaciones en sus características con el tipo denominado por él, Ante A y B, especialmente en el tratamiento de la superficie y temperante utilizado (1964, pp. 30, 31, 32).

El estudio sobre lo que hasta el momento se viene denominando Chanka, se encuentra en sus momentos iniciales. Excavaciones que en el futuro puedan hacerse, tanto en Naupas, Caballoyuq y en los otros sitios ubicados por Lumbreras, permitirán completar el conocimiento de un pueblo de singular importancia.

Las informaciones que nos proporcionan las fuentes documentales sobre los Chankas, unidas a las evidencias de la cultura material, pueden permitir en el futuro resolver el problema que plantea la demostración que si los Chankas que lucharon contra los ejércitos incaicos, fueron el mismo pueblo que vivió en el tipo de poblados como Naupas y son los productores de una cultura material rudimentaria a la cual se denomina actualmente "Chanka".

\section{APÉNDICE}

En la prospección superficial en el distrito de Vinchos, hemos registrado la existencia de varios sitios arqueológicos no estudiados hasta el momento y de los cuales damos noticia:

\section{1.- RITKRA:}

Situado al S.O. de Vinchos en donde se han ubicado una serie de abrigos rocosos con huesos humanos y fragmentos de cerámica de tipo Huarpa y Cruz Pata.

\section{2.- PUKRUPATA:}

Situado al N. del pueblo de Paqcha, donde existen construcciones derruidas que, al parecer, son chullpas incaicas que contienen huesos y cráneos humanos.

\section{3.- AYAPATA:}

Situado a $5 \mathrm{~km}$ al N.O. de Paqcha, donde se observan construcciones circulares de $6 \mathrm{~m}$ de diámetro, con muros de piedra de dos hileras unidos con barro de $0.50 \mathrm{~cm}$ de ancho.

Un segundo tipo de construcciones de forma rectangular con el mismo tipo de aparejo y además varias cuevas funerarias protegidas 
con muros que contienen huesos y cráneos de niños en gran cantidad.

Por último, se encuentran chullpas con muros de piedra unidos con barro, en igual forma que las construcciones circulares. Lo interesante son las cornisas de lajas grandes y la forma de las puertas que son trapezoidales y el dintel monolítico que son típicamente incaicos. En estas chullpas se observan también huesos y cráneos humanos deformados y con trepanación.

La cerámica que se encuentra no es incaica sino de tipo Arqalla, propia de los lugares Chankas.

\section{4.- WARIPIRQA:}

Ubicado muy cerca del lugar anterior, presenta estructuras cuadradas y circulares, edificada con los materiales y técnicas descritas.

La cerámica que se encuentra en este sitio, es de tipo Huarpa, Cruzpata, Caja, Inca y Arqalla.

\section{5.- MUYU URQU:}

Al Sur Oeste de Paqcha. Es un montículo donde existe una gran cantidad de variedad de restos cerámicos como Chavinoide negro, tipo Wichqana; Tunasniyuq, Rancha, Huarpa, Caja, Ocros, diversos fragmentos Wari, Arqalla, Inca y contemporánea.

Por otra parte, se recogieron algunos restos líticos como raspadores, puntas de proyectil, hachas con hombros y otros artefactos.

\section{BIBLIOGRAFÍA}

BONAVIA, Duccio

"Caballoyuq. Investigaciones arqueológicas en la ceja de selva de Ayacucho". Lima. Arqueológicas № 6. Museo Nacional de Antropología. 1964.

\section{CHAHUD, Carlos}

"Investigaciones arqueológicas en Vilcashuamán”- Huamanga. Informe presentado a la Universidad Nacional San Cristóbal de Huamanga. 1965.

\section{GONZÁLEZ CARRÉ, J. E.}

"Investigaciones arqueológicas e Nawimpukio". Huamanga. Informe presentado a la Universidad Nacional San Cristóbal de Huamanga. 1965.

\section{LUMBRERAS, Luis}

"Sobre los Chancas". Lima. Acta del II Congreso de Historia del Perú. Vol. I. pp. 211-242. 1958. 